des mondes, Table ronde organisée par le CNRS, Paris et la G.W. Leibniz-Gesellschaft, publié par A. Heinekamp, A. Robinet. Stuttgart, S. 47-64.

König, S. 1751. De universali principio aequilibrii et motus in vi viva reperto, deque nexu inter vim vivam et actionem, utriusque minimo, dissertatio. Nova Acta Eruditorum, S. 125-135, 162-176. Ich zitiere den Wiederabdruck in: L. Euler, Opera omnia, Bd. II,5, hrsg. v. J.O. Fleckenstein. Lausanne 1957, S. 303-324.

Maupertuis, P.M. de. 1740. Loi du repos des corps. Mémoires de l'académie des sciences de Paris, S. 170-176. Ich zitiere den Wiederabdruck in: L. Euler, Opera omnia, Bd. II, 5, S. 268-273.

derselbe. 1744. Accord de différentes loix de la nature. Mémoires de l'académie des sciences de Paris, S. 417-426. Ich zitiere den Wiederabdruck in: L. Euler, Opera omnia, Bd. II, 5. S. 274-281.

derselbe. 1746. Les loix du mouvement et du repos déduites d'un principe métaphysique. Mémoires de l'academie des sciences de Berlin, S. 267-294. Ich zitiere den Wiederabdruck in: L. Euler, Opera omnia, Bd. II, 5, S. 282-302.

Planck, M. 1915. Das Prinzip der kleinsten Wirkung. In: Die Kultur der Gegenwart, hrsg. v. Paul
Hinneberg, 3. T., 3. Abt. u. d. Leitung v. E. Lecher, 3. Bd., Leipzig - Berlin, S. 692-702. Ich zitiere den Wiederabdruck in: Physikalische Rundblicke. Gesammelte Reden und Aufsätze. Leipzig 1922, S. 103-119.

Pulte, H. 1989. Das Prinzip der kleinsten Wirkung und die Kraftkonzeption der rationalen Mechanik. Eine Untersuchung zur Grundlegungsproblematik bei Leonhard Euler, Pierre Louis Moreau de Maupertuis und Joseph Louis Lagrange. Stuttgart.

Rosenbloom, P. 1950. The Elements of Mathematical Logic. New York.

Schramm, M. 1985. Natur ohne Sinn? Das Ende des teleologischen Weltbildes. Graz - Wien - Köln.

Szabó, I. 1987. Geschichte der mechanischen Prinzipien und ihrer wichtigsten Anwendungen. 3. Aufl. Basel - Boston - Stuttgart.

Thiele, R. 1982. Leonhard Euler. Leipzig.

Tuffet, J. (Hrsg.). 1967. Voltaire, Histoire du Docteur Akakia et du natif de St. Malo. Paris.

Anschrift des Autors:

Prof. Dr. Eberhard Knobloch

TU Berlin Sekr. TEL 2

Ernst-Reuter-Platz 7

10587 Berlin

\title{
Die verschiedenen Phasen der Lehreraus- und -fortbildung Gibt es eine Gesamtkonzeption?
}

\section{von Günter Schmidt}

Beim ersten Zugang wird man die im Thema gestellte Frage natürlich mit „Ja“ beantworten. Ein Blick in die im Prinzip übereinstimmenden Ausbildungsverordnungen der verschiedenen Bundesländer zeigt von der Struktur her eine Geamtkonzeption. Diese besteht für das Lehramt an Gymnasien zunächst im Modell der Zweiphasigkeit. Die 1. Phase (Studium an der Universität)ist im wesentlichen auf die wissenschaftliche Ausbildung im allgemeinen in zwei Fächern und einen ergänzenden (additiven?) Teil in erziehungswissenschaftlichen Studien ausgerichtet. Sie dauert in der Regel 10-12 Semester und schließt mit dem 1. Staatsexamen. Mit unterschiedlichem (insgesamt jedoch relativ geringem) Gewicht umfaßt diese 1. Phase auch fachdidaktische Studien. Die 2. Phase (Referendariat) versteht sich als reflektierte und gleichzeitig praxisorientierte Berufseinführungsphase, in der Fachdidaktik und-methodik (in 2 Fächern) im komplexen Umfeld pädagogischen Denkens und Handelns vermittelt werden. Sie dauert 2 Jahre und schließt mit dem 2. Staatsexamen ab.

Soweit die von der Struktur her vermittelte und seit vielen Jahrzehnten im wesentlichen unveränderte Konzeption. Im Inneren haben selbstverständlich die vielen pädagogischen und bildungspolitischen Reformen auch für die Gymnasiallehrerausbildung Spuren hinterlassen. Dabei wird es schon schwieriger, diese wechselnden Spuren in eine Konzeption einzuordnen.

Viele Untersuchungen und Veränderungs- bzw. Verbesserungsvorschläge zur Lehrerausbildung beziehen sich jeweils auf eine spezielle Phase dieser Ausbildung mit den Schwerpunkten der 1. oder 2. Pha- se. Nach meiner Auffassung wäre es äußerst nützlich, den Blick mehr auf das ganze zu lenken, nämlich auf das Zusammenwirken und die Verbindungen der verschiedenen Phasen, wobei ich hierbei auch die vorbereitende Phase der eigenen Schülererfahrungen des angehenden Mathematiklehrers (0. Phase) und die berufsbegleitende 3. Phase der Lehrerfort- und -weiterbildung (lebenslanges Lernen!) ausdrücklich mit einschließen möchte. Dabei wird man schnell auf mangelnde Abstimmungen, auf fehlende Beziehungen und sogar auf deutliche Brüche aufmerksam, die 
zu einem großen Teil die längst als notwendig erkannten Veränderungen von Einstellungen, Kompetenzen und Verhaltensweisen von LehrerInnen behindern oder ihnen sogar entgegenwirken. Wirksame Reformen müssen von einer stimmigen Gesamtkonzeption der verschiedenen Phasen ausgehen und dabei noch zusätzlich die (sich verändernden) institutionellen und soziokulturellen Bedingungen der realen Schule einbeziehen.

Ich will mich zunächst kritisch mit Problemen und Defiziten in den verschiedenen Phasen der Lehrerausbildung in Mathematik auseinandersetzen, in dem hier gegebenen Zusammenhang mit einem Schwerpunkt im Blick auf die 1. Phase. Diese wird in der hier gebotenen Kürze notwendigerweise in Teilen zu pauschal und zu wenig differenziert sein, aber damit die nach meinen Erfahrungen wichtigen Probleme mit der notwendigen Pointierung herausstellen. In dem anschließenden Punkt „Lösungsansätze“ werde ich versuchen, die von verschiedener Seite aus vorhandenen vielfältigen Versuche zur Überwindung der beschriebenen Defizite in eine optimistische und konstruktive Aufforderung einzubeziehen.

\section{Probleme und Defizite in den verschie- denen Phasen}

\section{Isolation der verschiedenen Phasen der Leh- rerausbildung}

Aus der Sicht vieler Lehrerinnen und Lehrer besteht häufig nur wenig oder überhaupt keine Verbindung zwischen den verschiedenen Phasen der Lehrerausbildung.

$\mathrm{Zu}$ den in der eigenen Schulzeit gewonnenen Erfahrungen und Zielvorstellungen finden die Lehramtsstudenten in ihrem wissenschaftlichen Studium an der Universität i.a. wenig Anknüpfungspunkte. Sie sind im wesentlichen damit beschäftigt, in den verschiedenen Fachgebieten der Fachwissenschaft Mathematik das geforderte hohe Spezial-Niveau zu erreichen und in den entsprechenden Prüfungen nachzuweisen. Dabei werden ihnen von Beginn an die Teildisziplinen in einem fertigen und systematischen Aufbau angeboten, von Prozessen der Begriffsentwicklung, von Steigerungen des Abstraktions- und Präzisierungsniveaus oder einer Reflexion des dargebotenen Aufbaus erleben sie nur wenig. Ein solches Erleben und Reflektieren ist aber eine wesentliche Bedingung für die angestrebte Profession des Lehrens und Vermittelns von Mathematik im Rahmen einer Bildungs- und Erziehungskonzeption und dies kann nicht ohne weiteres im Anschluß an ein fünfjähriges Studium nachgeliefert (aufgesetzt) werden.

Die oft verhängnisvolle Folge dieser Isolation wird dann in der 2. Phase der Ausbildung am Studiense- minar offensichtlich. Die hier vermittelten Konzeptionen von Mathematiklernen und die daraus abgeleiteten Formen des Lehrens und Vermittelns werden zwar als sinnvoll akzeptiert und angestrebt, es fehlt aber die breitere Basis des eigenen Erlebens und der daraus erwachsenen Einstellungen und Kompetenzen. Das erworbene Spezialwissen und -können ist höchstens im Sinne einer notwendigen Voraussetzung verwertbar. Nützlich erscheint dann häufig der Rückgriff auf die eigenen Schulerfahrungen als Schülerin oder Schüler oder das Einholen von und die Anpassung an überlieferte Routinen des Schulalltags. Die konstruktiv-kritische Auseinandersetzung mit verschiedenen Konzeptionen und der Erwerb eines eigenständigen Profils und eines verantwortungsvollen Mitgestaltungswillens weicht oft dem Wunsch nach wirkungsvollen Rezepten und Handlungsmustern. Dabei bieten dann die nach bewährtem $\mathrm{Mu}$ ster aufgebauten Schulbücher und aus dem Schulalltag abgeleitete Erfolgsrezepte weit stärkere Hilfen als die offenen, konzeptionell begründeten Abhandlungen und Vorschläge der mehr professionellen Fachdidaktik.

In dem auf die Referendarzeit folgenden Schulalltag wird die zuletzt beschriebene Verhaltensweise dann häufig noch verstärkt. Eben weil in den beiden überwiegend beziehungslosen Phasen der Lehrerausbildung kein kohärentes Bild und keine stabilen Kompetenzen für die Profession des Mathematiklehrens erworben wurden, verläßt man sich nun auf die im allgemeinen nicht stark reflektierte Erfahrung aus der eigenen und der beobachtbaren Praxis. In diesem Rahmen hat dann die Auseinandersetzung mit wandelnden Zielvorstellungen, mit fachdidaktischen Konzeptionen und pädagogisch eingebunden Methodenkonzeptionen weit weniger Chancen als die unmittelbare Verwertung von „bewährten“ Lehrstrategien, wie sie von den gängigen Lehrbüchern oder anderen unmittelbar umsetzbaren Materialien angeboten werden. Diese Aussagen können aus verschiedenen Quellen belegt werden, einmal durch das Ignorieren eines Großteils der Lehrerschaft von fachdidaktischen Zeitschriften, Tagungen oder Fortbildungsangeboten, zum anderen aber auch in der Bewertung der Schulbehörden: fachdidaktisches Engagement und methodische Kompetenzen sind in viel geringerem Maße beförderungsrelevant als etwa die Bewährung bei organisatorischen und außerunterrichtlichen Aktivitäten.

Als Fazit dieser beschriebenen Isolationen bleibt die Feststellung, daß es trotz des enorm hohen zeitlichen Aufwandes bzgl. der Lehrerausbildung nur unzureichend gelingt, wirklich konstruktiv-kritische Einstellungen und stabile Kompetenzen für die angestrebte Profession des allgemeinbildenden Fachunterrichts zu erreichen. 


\section{Defizite in den verschiedenen Phasen der Aus- bildung}

Es sollen hier im Schwerpunkt die inhaltlichen und auch methodischen Defizite genannt werden, die insbesondere die Ausgestaltung eines bildenden und erzieherischen Mathematikunterrichts erschweren.

Für die 1. Phase kann dies aus der Sicht der 2. Phase recht klar geschehen.

Es ist gute und bewährte Tradition, daß angehende Mathematiklehrerinnen und -lehrer eine gründliche und anspruchsvolle fachwissenschaftliche Ausbildung an der Universität erfahren. Für die Einlösung des mit ihrem Fachunterricht verbundenen Bildungsund Erziehungsanspruchs ist es wichtig, daß diese Fachausbildung sich in der Begegnung und Auseinandersetzung mit forschenden und lehrenden Mathematikerinnen und Mathematikern vollzieht und nicht $\mathrm{zu}$ früh auf die Bereiche und Methoden der gerade aktuellen Schulmathematik eingeengt wird.

Im Laufe der zunehmenden Komplexität von Schule und Unterricht und den damit wachsenden Ansprüchen an Kompetenz und Professionalität der Lehrenden kann sich aber auch die 1. Phase nicht ausschließlich auf den Aufbau spezieller fachlicher Kompetenz beschränken. Das heißt aber, daß bereits die 1. Phase der Lehrerausbildung in deutlichem Maße berufsbezogen ausgerichtet sein muß, d. h. auch auf Fähigkeiten und Kompetenzen des Vermittelns von mathematischer (Allgemein-) Bildung und damit verbundenen pädagogischen Intentionen. Um Kriterien und Hinweise für eine solch verbesserte Ausrichtung $\mathrm{zu}$ erhalten, ist es nützlich, sich Einseitigkeiten und Defizite des Fachstudiums im Hinblick auf die angestrebte Profession zu vergegenwärtigen.

- Da ist zuerst das einseitige Bild von Mathematik zu nennen, das der Lehramtsstudent im Mathematikstudium erwirbt bzw. vermittelt bekommt. Einseitigkeit hat dabei sowohl eine inhaltliche als auch eine wissenschaftstheoretische und methodische Komponente. Entscheidend ist, daß sich dem Student die Wissenschaft Mathematik im wesentliches als fertiges, geschlossenes System darstellt und auch so geboten wird. Entwicklungsprozesse (einschließlich bedeutender Irrtümer und Irrwege) fehlen ebenso wie Beziehungen von Mathematik zu anderen Wissenschaften, zu Anwendungsbereichen und zur Wirklichkeit. Jede Teildisziplin wird selbst in den Seminaren möglichst lückenlos und systematisch (und dann nur längs eines speziellen Pfades) von der Wurzel zur Krone aufgebaut. Dies ist zweifellos eine wichtige Sicht von Mathematik. Die andere, lebendige Sicht, nämlich der Weg vom Problem zum System, von einem Ausschnitt der schillernden Krone zu den Wurzeln wird nicht erfahren. Damit bleiben auch die Prozesse, die Irrtümer und Umwege und die vielfälti- gen Bezüge zwischen den Teildisziplinen und die fächerübergreifenden Einordnungen außen vor. Dieses Bild von Mathematik wird im Laufe des Studiums dann adaptiert und als übergeordnete Werteinschätzung übernommen. Für das Lernen und Lehren von Mathematik im Rahmen der auf Allgemeinbildung und erzieherisch ausgerichteten Schule bedeutet dies nicht nur eine Einschränkung, sondern häufig auch eine nicht geringe Barriere!

- Ebenso bedeutend sind die inhaltlichen Defizite und Einseitigkeiten. Trotz aller gegenteiligen Analysen und Forderungen und trotz ermutigender Einzelinitiativen an manchen Universitäten gehört die Geometrie zu den vernachlässigten Disziplinen in der Hochschulausbildung. Damit findet der sich verstärkende Kreislauf des Rückzugs der Geometrie aus der Schule seine wesentliche Verstärkung. Ähnlich ist es um die Stochastik bestellt: obwohl dieser Bereich seit über 20 Jahren mit guten Gründen in die Lehrpläne des allgemeinbildenden Mathematikunterrichts aufgenommen wurde, gehört er noch immer nicht zum Pflichtteil der Universitätsausbildung. Ebenso vernachlässigt werden in der Breite nach wie vor wichtige Erkenntnisse und Prozesse aus der Geschichte der Mathematik und die Verbindungen von Mathematik zu anderen Fächern und zu Anwendungsbereichen. Die in der 2. Phase nun deutlich gewichteten Anforderungen an den Lehrer bedeuten auf Grund nicht erworbener Kompetenzen in diesen Bereichen dann allzuoft eine Überforderung.

- Eng verbunden mit den eben genannten inhaltlichen Defiziten sind die wenig vielfältigen methodischen Erfahrungen der Studenten. Nach wie vor ist die Vorlesung mit ihrem fast auschließlichen Mitteilungscharakter und der Aufforderung nach passiver Übernahme von fertigen Konzepten vorherrschend. Stärker selbstverantwortliche Lernstrategien mit höherer Eigentätigkeit und (nach-) entdeckendem Lernen bleiben zu lange außen vor. Als Medium der Vermittlung hat die Tafel unbestrittenes Monopol, mit ihrer Unterstützung wird Wissen linear, sequentiell und verbal-unanschaulich vermittelt. Ohne die grundsätzliche Eignung dieses Mediums gerade für die Mathematik in Frage zu stellen, so bedeutet die ausschließliche Beschränkung hierauf doch den Verzicht auf weitere Chancen. So spielt zum Beispiel der in anderen Bereichen längst selbstverständliche Einsatz des Computers als Veranschaulichungsmedium oder als Werkzeug gerade in Mathematik noch keine angemessene Rolle. Die selbständigen Aktivitäten der Studenten richten sich auf Grund der verpflichtenden Prüfungen dann auf eine möglichst effiziente Wissensübernahme, weniger auf das reflektierende Durchdringen und das vernetzte Einordnen der Einzelkenntnisse. Selbst Proseminare und Seminare sind meist nach gleichem Muster ausgerichtet, wirkli- 
che Kommunikation und vertiefte Reflexion und Diskussion findet auch hier zu selten statt.

- Als letztes, aber in der Bedeutung nicht geringes Defizit muß schließlich noch die zu geringe Orientierungshilfe erwähnt werden, die das Universitätsstudium für Eignung und Ausrichtung auf die angestrebte Profession des Vermittlers und Erziehers anbietet. Der Gymnasiallehrer wird in seiner 1. Ausbildungsphase nur mit fachorientierten Anforderungen konfrontiert, nach selbigen erfolgt auch die Auslese und überwiegend auch die Entwicklung des Selbstwertgefühls. Die eingestreuten zwei- bis vierwöchigen Schulpraktika bieten keine wirklichen Orientierungshilfen oder Bewährungsproben, ebensowenig die vereinzelten mehr laborartigen Veranstaltungen im Rahmen der Fachdidaktik. Damit wird die ganze Last der beruflichen Bewährung auf die zweijährige Referendarzeit verschoben, die aber in der Lebensplanung der nunmehr 25 bis 30 -jährigen keine wirkliche Alternative zuläßt.

Die Probleme und Defizite der 2. Phase der Lehrerausbildung sind zwangsläufig mit denen der 1 . Phase verbunden. In meiner beruflichen Praxis erlebe ich sie überwiegend als Widersprüche und Reibungen zwischen Zielabsichten und Realisierungsmöglichkeiten.

Vom Ziel her wird ein Mathematiklehrer gestützt, der Lernen nicht als möglichst effektive Übernahme von Wissen vom Lehrer begreift, sondern mehr als eigenständige, aktive Übernahme von Wissen, Einstellungen, Fähigkeiten und Strategien von seiten des Lerners. Die komplexen Aufgaben des Lehrers bestehen dabei im Initiieren, Moderieren und kompetenten Stützen der Lernprozesse. Dies ist die heutige in der pädagogischen Ausbildung vertretene Konzeption und Wunschvorstellung. Sie zielt auf eigenverantwortliche Gestaltung von Unterricht in offenen Unterrichts- und Interaktionsformen, sie müßte den Lehramtsanwärter als kompetenten Partner im Ausbildungs- und Beratungsprozess begreifen. Reale Unterrichtspraxis, institutionelle und bürokratische Vorgaben und enge Beurteilungskriterien und -rituale (z. B. 45-minütige Lehrproben als zentrale Ausbildungsveranstaltung oder die einstellungsrelevante „Beurteilung“ solch komplexer Kompetenz durch fragwürdige Mittelwerte mit zwei Stellen hinter dem Komma) begünstigen demgegenüber noch immer häufig möglichst gute Anpassungsleistungen der Lehramtsanwärter an bestehende Verhältnisse. Dieser Widerspruch ist schwer aufzulösen, solange von der 1. Phase zuwenig Kompetenzen aufgebaut werden und die 2. Phase in der Folge sich dann noch immer weithin an das Vorbild der „Meisterlehre“ anlehnt.

Dies hat dann auch Konsequenzen für die 3. Phase der Fort- und Weiterbildung. Vom Idealbild her müßte die zweite Phase mit reflektierter Praxis auf Kompetenzen und Einstellungen zum „lebenslangen Lernen" und zur aktiven Mitgestaltung und Weiterentwicklung von Unterricht hinführen. In der Realität bedeutet sie in vielen Fällen noch den Abschluß des Lernens. Der dann praktizierenden Lehrerinnen und Lehrer verstehen sich kaum noch als Mitgestalter an didaktischen Forschungen und unterrichtlichen Reformvorhaben, mehr als Ausführende vorgegebener Lehrpläne und Konzeptionen von Unterricht. Dem entspricht weitgehend auch die institutionell vorherrschende Auffassung von der Aufgabe der Lehrenden, die durch hohe Unterrichtsverpflichtung und defensive Handhabung von Fort- und Weiterbildungsmöglichkeiten gekennzeichnet ist.

\section{Lösungsansätze}

In den Aussagen des vorhergehenden Abschnitts wurde auf vorhandene Probleme und Defizite der Lehrerausbildung hingewiesen. Damit wurde in gewisser Pauschalisierung ein recht negatives Bild gezeichnet, in dem durchaus vorhandene Reformansätze und Verbesserungsbemühungen zunächst keine Erwähnung fanden. Selbstverständlich gibt es diese, überall und in vielen individuellen Ausprägungen. Ohne solch positive Erfahrungen wäre auch die Beschreibung der Defizite kaum möglich.

In diesem letzten Abschnitt sollen nun mögliche und in vielen Einzelfällen bereits realisierte Verbesserungschancen genannt werden, verbunden mit der Forderung nach einer breiteren konzeptionellen Berücksichtigung.

Um auch hier möglichst konkret und konstruktiv vorzugehen, beginne ich mit einigen Projekten aus meinem eigenen Erfahrungsbereich, daran anschließend werden allgemeinere Perspektiven und Forderungen zur stärkeren Berücksichtigung einer wünschenswerten Gesamtkonzeption skizziert.

\section{Modellierungswoche für SchülerInnen und LehrerInnen}

In Zusammenarbeit des Zentrums für Praktische Mathematik (Universität Kaiserslautern und Universität Darmstadt) und den Kultusministerien der Länder Hessen und Rheinland-Pfalz wurden im Juni 1993 und im Juni 1994 jeweils ein recht aufwendiges Projekt erfolgreich durchgeführt, das in Anlehnung an bewährte Veranstaltungen mit StudentInnen als "Modellierungswoche“ bezeichnet wurde. ([10]) Hier beschäftigten sich 40 begabte und an Mathematik interessierte SchülerInnen (12. Klasse) und 16 LehrerInnen eine Woche lang mit mathematischen Anwendungsproblemen. Das Besondere hieran war ein- 
mal die Arbeitsform und zum anderen die inhaltliche Betonung des Modellierens. Es wurden jeweils Gruppen/Teams von je 5 SchülerInnen und 2 LehrerInnen gebildet, denen von BetreuerInnen der Universität ein spezielles Problem aus einem bestimmten Anwendungsbereich vorgelegt wurde, das dann von der Gruppe mit Hilfe mathematischer Modelle gelöst werden sollte. Die Lösungsprozesse und Ergebnisse wurden dann am letzten Tag der Gesamtgruppe in allgemeinverständlicher Form präsentiert. Interessant waren dabei in gleichem Maße die Lern- und Kommunikationsprozesse in den Gruppen. Es war nicht klar, welche Mathematik zur Lösung des Problems herangezogen werden mußte. Bei allen Problemen stellte sich heraus, daß man auf verschiedene mathematische Disziplinen zurückgreifen mußte, zum Teil mußten Kenntnisse neu erworben oder erweitert werden. Die LehrerInnen waren in die Arbeit gleichberechtigt und partnerschaftlich einbezogen, sie kannten nicht die Antworten im voraus und konnten ebenso wie die SchülerInnen bestimmte Kenntnisse und Fähigkeiten aus der Mathematik und auch aus anderen Fachbezügen in die Teamarbeit einbringen. Phasen großer Schwierigkeiten wechselten mit Phasen des Erfolges ab. Mit großer Selbstverständlichkeit wurde der Computer als Werkzeug eingesetzt, entweder zur anschaulichen Präsentation von Ergebnissen oder zur Realisierung von Lösungsansätzen. Die Schilderung kann hier nicht weiter detailliert werden, in dem hier gegebenen Zusammenhang soll nur ein ermutigendes Fazit festgehalten werden: Viele der oben genannten Einseitigkeiten im Bild von Mathematik und in den Vermittlungsmethoden konnten hier durch exemplarisches Erleben und Handeln aufgelöst und durch ein reicheres Spektrum ergänzt werden. Die Übertragung solcher Projekte in passend variierter Form in den Unterricht an Schulen, in die Ausbildung der StudentInnen an der Universität (Modellierungsseminare) oder der ReferendarInnen im Studienseminar (Projekte) eröffnet Perspektiven zur Überwindung vieler der genannten Defizite. Natürlich liegen noch keine erprobten Konzepte vor, aber hier dürfte sich eine konzertierte Aktion von FachwissenschaftlerInnen, DidaktikerInnen, LehrerInnen und SchülerInnen lohnen.

\section{Fachdidaktik-Seminare mit übergeordneten Leitthemen}

Ich will hier in der gebotenen Kürze einige ermutigende Erfahrungen beschreiben, die ich im Rahmen meines Lehrauftrags für Fachdidaktik an der Universität Mainz sammeln konnte. Aus oben genannten Gründen bin ich von themenbezogenen Seminaren zur Didaktik der Algebra, Geometrie, Analysis, Stochastik u. a. (die nach wie vor eine wichti- ge und sinnvolle Ergänzung/Erweiterung der entsprechenden Fachvorlesungen darstellen) zunehmend zur Orientierung an bestimmten Leitthemen und didaktischen Prinzipien übergegangen. Als Beispiele seien genannt:

- Problemlösen im Mathematikunterricht

- Modellbilden im Mathematikunterricht

- Experimentelle Mathematik

- Mathematik und Wirklichkeit

- Entdeckendes Lernen im Mathematikunterricht

Dabei wurde den StudentInnen kein eng begrenzter Seminarvortrag angeboten, sondern mehr die Bearbeitung von bestimmten Aspekten des Themas an Hand konkreter Vorschläge in der fachdidaktischen Literatur. Diese Bearbeitung erfolgte in Gruppen von jeweils 2-3 StudentInnen mit dem Ziel der möglichst informativen und attraktiven Gestaltung von Seminarsitzungen. Dabei sollte diese Gestaltung auf die gegebene Adressatengruppe der KommilitonInnen (StudentInnen ab 5. Semester) bezogen sein und war dann neben den Inhalten auch Gegenstand anschließender Reflexionen und Diskussionen. Die erfreulichen und ermutigenden Erfahrungen mit diesem Ansatz bestehen einmal in der intensiven Auseindersetzung mit mathematischen Inhalten mit gebietsund fächerübergreifenden Aspekten, zum anderen in der (äußerst kreativen) methodischen Ausgestaltung der Darbietung in medienunterstützten Vortragsformen sowie in aktiven Interaktionsformen mit eingestreuten Aktivitätsphasen, Gruppenarbeiten und Diskussionen. Dabei traten häufig auch reflektierende Rückbezüge auf Themen der Standardvorlesungen auf, nicht selten mit Rückfragen und eingeforderten Statements der jeweiligen Dozenten dieser Vorlesungen. Beteiligung und Engagement der StudentInnen ging weit über die Pflichtforderung hinaus, nach deren Aussage wesentlich auf Grund der erkennbaren Vielseitigkeit des fachlichen Spektrums und des deutlichen Bezugs zur angestrebten Profession der Vermittlung mathematischer Bildung. Es scheint lohnend, auch Annäherungen oder Variationen solcher Ansätze von der fachbezogenen Ausbildung (Seminare/Projekte) her zu versuchen.

\section{Produktorientierte Lehrerfortbildung}

Als letztes Beispiel soll eine Form der Lehrerfortbildung beschrieben werden, die auf stärkere Mitbeteiligung an der didaktischen Entwicklung und die eigenverantwortliche Gestaltung von Reformvorhaben ausgerichtet ist. Gemeint sind hier nicht die gängigen Veranstaltungen, in denen Didaktiker oder Kommissionen ihre Konzepte zu bestimmten Fragen und Themen des Mathematikunterrichts in Vorträgen vorstellen, sondern wo kleinere, regionale Gruppen 
von Lehrerinnen und Lehrern eigene Konzepte entwickeln, diskutieren und mit unterrichtlicher Erprobung schließlich in Materialien und Handreichungen zusammenfassen und damit einem erweiterten Kreis von Interessenten zugänglich machen. Durch solche didaktische Forschungsarbeit im Kleinen wird automatisch eine Kooperation und Kommunikation auch mit professionellen Didaktikern und Fachwissenschaftlern und die damit verbundene Auseinandersetzung mit fachdidaktischer Literatur angeregt, dies nicht im Sinne des Konsumenten, sondern des Mitgestalters mit der wichtigen Kompetenz des Praktikers. Hiermit könnte auch die leider vorhandene Kluft zwischen professioneller Fachdidaktik und Schulpraxis zum Nutzen beider Seiten verringert werden. Einzelaktivitäten dieser Art gibt es erfreulicherweise viele, es gilt hier die Basis zu verbreitern. Voraussetzung hierfür ist einmal die Vorbereitung durch bewußte Entwicklung von Kompetenzen und Bereitschaften über die Ausbildungsphasen und nicht zuletzt die notwendige Wertschätzung solcher Ansätze von den rahmengebenden Institutionen.

\section{Einige allgemeinere Perspektiven und Forde- rungen}

Ich bin mir im klaren darüber, daß die hier aufgezählten wenigen Anätze nur im Rahmen einer Gesamtkonzeption in den verschiedenen Phasen der Lehrerausbildung zu der notwendigen Verbesserung beitragen können. Sie weisen aber in die Richtung, in der vorhandene Isolationen und Einseitigkeiten überwunden werden können. Für diese Richtung möchte ich zum Abschluß noch einige allgemeinere Perspektiven und Forderungen festhalten, die man in diesem Kreise durchaus als Handlungsaufforderung verstehen sollte.

Zentrale Grundlage für die Konzeption einer Mathematiklehrerausbildung ist die Konzeption (Vision) des Mathematikunterrichts. Ich berufe mich hier auf die Visionen eines anschaulichen und lebendigen Mathematikunterrichts, wie sie von Mathematikern und Didaktikern wie Georg Polya [7], Hans Freudenthal [8], Alexander Israel Wittenberg, Otto Toeplitz, Heinrich Winter, Erich Wittmann und vielen anderen überzeugend beschrieben und begründet werden. In jüngster Zeit finden deren Auffassungen ja auch Berücksichtigung in einer Auseinandersetzung der DMV um das (öffentliche) Bild der Mathematik $([4],[5])$. Ich will dies noch um eine Darstellung der „Entwicklungslinien Mathematikunterricht" ergänzen, in der die mehr dynamischen Zielrichtungen des Mathematikunterrichts den mehr traditionellen statischen Auffassungen gegenübergestellt werden.

\section{Entwicklungslinien Mathematikunter- richt}

- visualisiertes Lernen vs sprachlich-symbolhaftes Lernen

- entdeckendes, explorierendes Lernen, offenes Konzept vs statisch, linear-systemhaft, Fertigprodukt, geschlossenes Konzept

- handelndes, produktorientiertes Lernen vs beschreibend, analysierend, aufnehmend

- Herausstellen und Nutzen von Querverbindungen, gebiets- und fächerübergreifenden Aspekten („horizontales Lernen“, „modularer Aufbau“) vs Lernen in abgegrenzten Disziplinen „Schubladenlernen“ („vertikales Lernen“, „linearer Aufbau“)

- aktives Modellieren als Prozeß vs Darbieten von Modellen

- Ästhetisches Erleben (emotionale Komponente) vs Beschränkung auf analytisches, kalkülmäßiges Erschließen (kognitive Komponente)

- Orientieren an Leitbegriffen, Fundamentalen Ideen (Funktion, Iteration Linearität - Komplexität ... ) vs Orientierung an Fachdisziplinen Systematik

Aus diesen Darstellungen komme ich zu einer 1. These:

- Eine verbesserte Mathematiklehrerausbildung setzt einen verbesserten Mathematikunterricht voraus. Dies meint vor allem eine stärkere Passung zwischen der allgemein als sinnvoll erkannten Konzeption von Mathematikunterricht und der real erlebten und ausgeführten Unterrichtspraxis.

Dies erinnert auf den ersten Blick ein wenig an das Problem mit dem „Ei und dem Huhn“, weist aber bei genauerer Betrachtung durchaus auf Konsequenzen hin. In 2. 1 habe ich schon auf den beträchtlichen Einfluß hingewiesen, den die 0. Phase (der erlebte Schulunterricht) als Bezugs- und „Fluchtrahmen“ in der Lehrerausbildung ausüben kann. Es gibt ja durchaus auch positive Erlebnisse im Vermitteln eines vielschichtigen Bildes von Mathematik gemäß den eben beschriebenen Visionen während der eigenen Schulzeit. Diese gilt es in der Phase des wissenschaftlichen Studiums aufzugreifen und weiterzuführen, d. h. es müssen neben den unverzichtbaren fachspezifischen Kenntnissen eben auch Kompetenzen bezüglich der Reflexion, der Heuristik und Problemlösefähigkeiten, der Anwendungen und des Zusammenhangswissens einschließlich fächerübergreifender Aspekte entwickelt werden. Dies bietet dann eine stabile Basis für die Ausformung einer eigenständigen und weniger routineanfälligen Unterrichtskompetenz in der 2. Phase, die wiederum den Willen und die Fähigkeit zur aktiven Mitgestaltung an der Entwicklung des Mathematikunterrichts in einer mit lebenslangem Lernen verbundenen Praxis fördern. Von daher läuft 
diese 1. These konsequent auf die Forderung der engeren Verzahnung der verschiedenen Phasen der Lehrerausbildung hinaus, eben auf eine möglichst stimmige und überzeugende Gesamtkonzeption.

Diese schöne und zugegebenermaßen recht allgemeine Forderung soll nun für die benannten Phasen 1 bis $3 \mathrm{zu}$ konkreteren Forderungen führen:

- Die 1. Phase der Lehrerausbildung muß in höherem Maße berufsbezogen ausgerichtet sein, d. h. auf Fähigkeiten und Kompetenzen des Vermittelns von mathematischer (Allgemein-) Bildung und damit verbundenen pädagogischen Intentionen.

Dies soll und kann nun keinesfalls durch Reduzierung der fachlichen Ausbildung und Ausweitung eines (beziehungslosen) pädagogischerziehungswissenschaftlichen Studiums geschehen. Es erfordert vielmehr eine Vertiefung des fachwissenschaftlichen Studiums in Richtung des Verstehens von Genese mathematischer Begriffe, der Zusammenhänge von Teildisziplinen, der Prozesse des Mathematiktuns und der Verbindung von Mathematik $\mathrm{zu}$ anderen Bereichen und der Wirklichkeit. Dies ist ein anspruchsvolles Ziel, das sicher nur auf Kosten mancher Spezialisierungen und des Umfangs mathematischer Spezialkenntnisse erreichbar ist. Es ist eben der notwendige Anspruch für ein kompetentes Lehren und Vermitteln von Mathematikunterricht, und dieser Anspruch ist sicher in manchen Teilen verschieden (nicht geringer) von dem speziellen Anspruch an den künftigen Anwender von Mathematik oder den wissenschaftlichen Forscher. Gefordert ist also die aktive Aneignung eines Bildes von Mathematik und damit verbundener Fähigkeiten, die ein eigenständiges, selbstbewußtes und flexibles Lehren begünstigen. Wie dies konkret aussehen kann, dafür bleibt mir an dieser Stelle nochmals der Verweis auf Vorschläge von Polya, Freudenthal u. a. oder auch der Hinweis auf die zukunftsweisende und leider noch viel zu wenig eingelöste Denkschrift der DMV zur „Ausbildung von Studierenden des gymnasialen Lehramts im Fach Mathematik" [1].

Zur Ergänzung noch eine Anmerkung zu der immer wieder geforderten Durchlässigkeit zwischen Lehramts- und Diplomstudiengang. In einer Zeit der knappen Arbeitsplätze ist diese besonders ernst zu nehmen. Ich glaube nicht, daß die geforderte „Berufsbezogenheit" im Fachstudium diese Durchlässigkeit verringert, zumal sie eben nicht eine additive Erhöhung isolierten erziehungswissenschaftlichen Studienanteils meint, sondern eine stärkere Berufsbezogenheit in der fachwissenschaftlichen Ausbildung. Die Konsequenzen betreffen ja eine inhaltliche Schwerpunktbildung auf schulrelevante Themen (selbstverständlich auf hohem Niveau mit exemplarischer Vertiefung zur Forschungskompetenz) und darüberhinaus eine methodische Profilierung von Lehrveranstaltungen, die auch für den Diplomstudiengang von Nutzen sein können (z. B. Zusammenhangswissen, Kenntnisse über Anwendungen, fächerübergreifende Einordnungen u. ä. ). Auch diese Auffassung wird in den erwähnten DMVEmpfehlungen von 1979 ausführlicher begründet und spielt auch in der gegenwärtigen Diskussion der DMV um das Bild von Mathematik eine bedeutende Rolle.

Damit komme ich zu einigen Aussagen und Thesen über die 2. Phase.

- Die zweite Phase muß als Berufseinführungsphase verstanden werden, in der die in der 1. Phase erworbenen Fach- und Urteilskompetenzen in den komplexen Zusammenhang praktischen pädagogischen Handelns eingebracht und in reflektierter Praxis vor allem zu einer verstärkten methodischen Kompetenz weiterentwickelt werden. Wichtig ist dabei: Sie beginnt nicht beim Stande Null, ReferendarInnen sind deshalb nicht als „Lehrlinge“ anzusehen, sondern als kompetente Partner in einem theoretisch reflektierten Erfahrungsprozeß.

Wenn diese Auffassung von AusbilderInnen konsequent vertreten und von den ReferendarInnen erlebt wird, lassen sich viele der häufig erlebten Probleme im Rollenverständnis und daraus resultierende Ängste und Negativeinstellungen vermeiden. Vor allem würde damit der wünschenswerte Typ des eigenständigen, Verantwortung wahrnehmenden Lehrers gefördert gegenüber einem mehr auf Anpassung und Ausführung von bestehenden Richtlinien bedachten "Unterrichtsingenieurs".

Allerdings erfordert diese Auffassung auch das Überdenken und Verändern mancher traditioneller Formen und Verordnungen in der Ausbildung der 2. Phase, wie ich sie oben schon erwähnt habe.

Hier soll nur noch eine These angefügt werden, die zur 3. Phase der Ausbildung überleitet.

- Die 2. Phase der Ausbildung darf nicht als Abschluß der Ausbildung verstanden werden, vielmehr als eine intensive Stufe im praxisorientierten Erwerb von Erfahrungen und Kompetenzen für die angestrebte Profession des Lehrers. Die Notwendigkeit (und der Nutzen) des lebenslangen Weiter- und Umlernens muß deutlich werden.

Damit zum Abschluß einige wichtige Forderungen für die 3. Phase:

- Lehrerfortbildung muß als eine elementare Verpflichtung in dem vielfältigen Arbeitsfeld des praktizierenden Lehrers verankert werden. Dies sowohl von der inneren Einstellung als auch von der äußeren Bereitstellung von Möglichkeiten und der Bewertung von seiten der Schulbehörden.

- Lehrerfortbildung muß in viel stärkerem Maße als bisher die LehrerInnen als aktiven und mitforschende Teilnehmer einbeziehen. Dies ist sowohl eine Aufforderung an die LehrerInnen als auch an die pro- 
fessionellen FachdidaktikerInnen und ErziehungswissenschaftlerInnen. Die in der Praxis erworbene Kompetenz und Erfahrung muß im gleichberechtigten Diskurs mit den theoretischen Konzeptionen verhandelt werden. Nur so läßt sich die gegenwärtig vorhandene Kluft zwischen professioneller Didaktik und gängiger Unterrichtspraxis wirkungsvoll verringern. Die Fachleiterinnen und Fachleiter können hier eine wichtige Mittlerfunktion einnehmen, dies in viel offensiverer und breiterer Weise als bisher. Die fachdidaktischen Zeitschriften könnten auf diese Weise ein wichtiges Medium werden, sie sind es gegenwärtig nur vereinzelt.

- Lehrerfortbildung muß neben der notwendigen Instruktion und Reflexion auch produktorientiert sein. Das heißt, aus Lehrerfortbildungsveranstaltungen müssen Materialien und Anschlußmaßnahmen entstehen, die in die Praxis hineinwirken! Hierfür gibt es ja ermutigende Beispiele, sie müssen aus der Vereinzelung zum verbreiteten Prinzip werden.

Die hier zuletzt vorgestellten Forderungen und Perspektiven enthalten einen gehörigen Schuß an Wunschvorstellungen. Alle Schritte in Richtung einer partiellen Realisierung müssen von einer Überwindung bestehender Defizite, Isolationen und Brüche zwischen den verschiedenen Phasen der Lehrerausbildung ausgehen. Damit schließe ich an die im Vortragsthema gestellte Frage an mit einer letzten Aufforderung: Wir brauchen eine stimmige und praktizierte Gesamtkonzeption für die Lehreraus- und fortbildung. Die fortgesetzte Arbeit aller Beteiligten von Universität und Schule hierfür ist herausfordernd und lohnend.

\section{Literatur}

[1] Deutsche Mathematiker-Vereinigung e. V. , DENKSCHRIFT zur Ausbildung von Studierenden des gymnasialen Lehramts im Fach Mathematik, Juli 1979

[2] Deutscher Verein zur Förderung des mathematischen und naturwissenschaftlichen Unterrichts e. V. , Empfehlungen zur Lehrerausbildung in Mathematik und den Naturwissenschaften, MNU 42/3, April 1989 [3] F. Schweiger, Zur mathematischen Ausbildung der Mathematiklehrer, in ZDM Heft 41992

[4] DMV-Mitteilungen 1993/94, Diskussion um Mathematik und Öffentlichkeit

[5] Heinrich Winter, Wie läßt sich Mathematikdidaktik als Hochschuldisziplin legitimieren?, in DMV Mitteilungen 2/94

[6] Fachdidaktische Überlegungen zur Referendarausbildung in Mathematik, in ZDM 1986, Heft 1 und 2

[7] Georg Polya, Mathematik und plausibles SchlieBen, Bd 1 und 2, 1975

[8] Hans Freudenthal, Mathematik als pädagogische Aufgabe, Bd 1 und 2, 1973

[9] Mies u. a. , Tendenzen und Probleme der Mathematiklehrerausbildung, in Schriftenreihe des IDM $6 / 1975$

[10] Zentrum für Praktische Mathematik, Die Modellierungswoche in der Pfalzakademie Lambrecht vom 20. -25. Juli 1993, Darmstadt/Kaiserslautern 1993

Dies ist die gekürzte und in Teilen geänderte Fassung eines Vortrags, den der Verfasser auf der Fachleitertagung im September 1994 in Fuldatal bei Kassel gehalten hat. Eine weitergehende Auseinandersetzung mit dem Thema erfolgt in dem Band 21 der IDM-Reihe „Untersuchungen zum Mathematikunterricht“ mit dem Titel „Mathematik allgemeinbildend unterrichten: Impulse für Lehrerbildung und Schule“ (Aulis, Köln, Herbst 1995).

Anschrift des Autors:

Günther Schmidt

Am Hahn 3

55442 Stromberg 